\title{
Distribuição de Gordura Corporal, Pressão Arterial e Níveis de Lipídios-Lipoproteínas Plasmáticas
}

\author{
Dartagnan Pinto Guedes, Joana Elisabete Ribeiro Pinto Guedes \\ Londrina, PR
}

\begin{abstract}
Objetivo - Investigar associações entre distribuição do tecido adiposo e níveis de pressão arterial e concentrações de lipídios-lipoproteínas plasmáticas, mediante controle de indicadores, quanto à quantidade de gordura corporal e à prática da atividade física.

Métodos - Estudo de 62 indivíduos com idades entre 20 e 45 anos. A distribuição do tecido adiposo foi determinada baseando-se na relação circunferência de cintural quadril (CCQ), e como indicador da quantidade de gordura corporal recorreu-se às informações do índice de massa corporal (IMC), enquanto o nível de prática da atividade física foi estabelecido mediante estimativas do consumo máximo de oxigênio ( $\mathrm{VO}_{2} \mathrm{max}$ ). As associações entre $C C Q$ e níveis de pressão arterial e de lipídios-lipoproteínas plasmáticas, com os efeitos do IMC e do $\mathrm{VO}_{2}$ max controlados estatisticamente, foram estabelecidas pelo coeficiente de correlação parcial.

Resultados - Após correção pelo IMC verificou-se significativa correlação parcial entre a distribuição centrípeta do tecido adiposo e os níveis de pressão arterial, LDL-C e triglicerídios plasmáticos. Entretanto, controlando-se $\mathrm{o} \mathrm{VO}_{2}$ max, não foram constatadas associações significativas entre CCQ e qualquer variável sangüinea e pressão arterial.

Conclusãa - A distribuição centrípeta do tecido adiposo, independente da quantidade de gordura corporal, foi relacionada com concentrações de lipídioslipoproteínas plasmáticas e níveis de pressão arterial em ambos os sexos. A prática da atividade física parece ser um importante modulador dessa associação, enfatizando seu papel no controle dos fatores de risco predisponentes às doenças cardiovasculares.
\end{abstract}

Palavras-chave: gordura corporal, atividade física, fatores de risco

\section{Body Fat Distribution, Blood Pressure and Plasma Lipids and Lipoprotein Levels}

Purpose - To study associations between FAT distribution and blood pressure levels and concentrations of lipids and lipoproteins, irrespective of body fat content and physical activity.

Methods - A sample of 62 subjects of both genders aging 20-45 years-old was used in the study. The adipose tissue distribution was based on the waist-to-hip ratio (WHR). Body fat content was estimated by the body mass index (BMI), and physical activity was assessed by maximal oxygen uptake $\left(\mathrm{VO}_{2}\right.$ max). Partial correlation analyses were used to determine the strength of the associations.

Results - After correction for BMI there was a significant partial correlation between centripetal adipose tissue distribution and serum triglyceride, LDL-cholesterol levels, and blood pressure. However, controlling for $\mathrm{VO}_{2}$ max, there was no significant association between WHR and any serum variable and blood pressure.

Conclusion - The centripetal adipose tissue distribution, independent of body fat content, was related to lipid and lipoprotein plasma concentrations and to blood pressure levels in both sexes. Physical activity seems to be an important modifier of this relationship, emphasizing its role in the control of cardiovascular risk factors.

Key-words: body fat, physical activity, risk factors

Arq Bras Cardiol, volume $70\left(n^{0} 2\right), 93-98,1998$

Centro de Educação Física e Desporto da Universidade Estadual de Londrina. Apoio do $\mathrm{CNPq}$

Correspondência: Dartagnan Pinto Guedes - Rua da Lapa 300 - 86015-060 Londrina, PR

Recebido para publicação em 2/9/97

Aceito em 20/11/97
Estudos recentes sugerem que a variação da distribuição anatômica da gordura corporal é importante indicador morfológico relacionado com complicações endócrinas e metabólicas predisponentes ao aparecimento e desenvolvimento de doenças cardiovasculares ${ }^{1-3}$. Indivíduos com 
essa disposição centrípeta da gordura corporal tendem a apresentar maior incidência de diabetes ${ }^{4,5}$, hipertensão ${ }^{6,7} \mathrm{e}$ alterações desfavoráveis no perfil das lipoproteínas plasmáticas $^{8-11}$.

A monitorização da quantidade de gordura corporal e da prática da atividade física tem recebido grande notoriedade em aspectos relacionados à promoção da saúde, não apenas por suas ações isoladas na prevenção e no controle das doenças cardiovasculares ${ }^{1,2,12,13}$, mas também, por induzirem alterações desejáveis em outros fatores de risco, sobretudo, nos níveis de lipídios plasmáticos e de pressão arterial (PA) ${ }^{6-8,10,11}$.

Diante desta perspectiva, a magnitude da relação entre maior acúmulo de gordura na região central do corpo e níveis de lipídios sangüíneos e de PA pode sofrer variações em função da quantidade de gordura corporal e do impacto da prática de atividade física. Na tentativa de contribuir para a elucidação do problema, o estudo procura analisar associações entre índices de distribuição anatômica da gordura corporal e níveis séricos de lipídios/lipoproteínas e de PA, mediante controle de indicadores voltados à quantidade de gordura corporal e à prática da atividade física.

\section{Métodos}

Foram estudados todos os funcionários do setor administrativo da Universidade Estadual de Londrina, que participavam dos programas de exercícios físicos oferecidos pelo Centro de Educação Física e Desporto daquela instituição, no $2^{\circ}$ semestre de 1995 e selecionados os que atendiam aos cinco critérios básicos: a) ter entre 20 e 45 anos de idade; b) estar participando dos programas de exercícios físicos há pelo menos 6 meses; c) ser não-fumante nos últimos 5 anos; d) não utilizar medicamentos anti-hipertensivos ou similares; e) não estar sendo submetido a dietas especiais, constituindo grupo de 62 sujeitos, 21 homens e 41 mulheres.

A dosagem dos lipídios plasmáticos foi realizada mediante a coleta de amostras de $10 \mathrm{ml}$ de sangue venoso na prega do cotovelo, após um período de 10-12h em jejum, entre 7:00 e 8:00h da manhã. O soro foi imediatamente separado por centrifugação, sendo determinados os teores de triglicerídios (TG), colesterol total (CT) e as frações, lipoproteínas de baixa densidade (LDL-C) e de alta densidade (HDL-C). Determinou-se o colesterol sérico total pelo método enzimático colesterol oxidase/peroxidase em aparelho espectofotômetro. O HDL-C foi medido pelo método reativo precipitante, e o LDL-C calculado pela fórmula de Friedewald ${ }^{14}$. Os triglicerídios séricos foram determinados pelo método enzimático glicerol.

Os níveis de pressão arterial sistólica (PAS) e diastólica (PAD) foram aferidos com auxílio de um esfigmomanômetro de coluna de mercúrio. Com o indivíduo sentado, após um período mínimo de 5 min de repouso, a PA foi medida no braço esquerdo. $\mathrm{O}$ valor da PAS correspondeu à fase I de Korotkoff e o da PAD à fase V, ou de desaparecimento dos sons. Foram realizadas duas medidas, sendo que o valor médio de ambas foi considerado para efeito de cálculo.
Estimativas quanto à quantidade e à distribuição da gordura corporal foram realizadas mediante o cálculo do índice de massa corporal (IMC) e da relação circunferência cintura/quadril (CCQ). O IMC foi calculado considerandose a razão entre o peso corporal e o quadrado da estatura $\left(\mathrm{kg} / \mathrm{m}^{2}\right)$. Para determinação da estatura, utilizou-se um estadiômetro com escala de medida de $0,1 \mathrm{~cm}$, enquanto para verificação do peso corporal foi empregada uma balança antropométrica com precisão de $100 \mathrm{~g}$, a partir do método apresentado por Gordon e col ${ }^{15}$. Quanto às circunferências de cintura e quadril, realizadas mediante utilização de uma fita antropométrica de aço flexível com precisão de uma casa decimal, recorreram-se às padronizações sugeridas por Callaway e col ${ }^{16}$.

O nível de prática da atividade física foi estabelecido mediante estimativas quanto ao consumo máximo de oxigênio ( $\mathrm{VO}_{2} \max$.). Para tanto, recorreu-se a teste de esforço de carga máxima em esteira rolante, de acordo com o protocolo de Bruce ${ }^{17}$. As indicações de suspensão do teste foram as recomendadas pelo Colégio Americano de Medicina do Esporte ${ }^{18}$. O tempo necessário para alcançar a freqüência cardíaca máxima teórica (220-idade) foi utilizado para as estimativas do consumo máximo de oxigênio ${ }^{19}$. Para aqueles avaliados que não foram capazes de alcançar sua freqüência cardíaca máxima teórica foi empregada, como referencial, a duração do teste de esforço.

O tratamento estatístico das informações foi realizado pelo pacote estatístico computadorizado Statistical Analysis System - versão 3.0 ${ }^{20}$. No primeiro momento, foram calculados os coeficientes de correlação simples de Pearson entre cada variável relacionada aos níveis de PA e de lipídio-lipoproteína plasmática e o IMC, CCQ e VO max. No momento seguinte, utilizando-se os procedimentos do coeficiente de correlação parcial, foram estabelecidas associações entre a CCQ e os níveis de PA e de lipídio-lipoproteína plasmática com os efeitos do IMC e do $\mathrm{VO}_{2} \max$ controlados estatisticamente.

\section{Resultados}

Informações estatísticas das variáveis analisadas são apresentadas na tabela I. No que se refere aos indicadores de gordura corporal, os valores médios encontrados apontam diferenças estatísticas favoráveis às mulheres nas duas variáveis consideradas. $\mathrm{O}$ índice de dimorfismo sexual foi mais elevado na CCQ (13\%), seguido do IMC (11\%). Quanto ao $\mathrm{VO}_{2}$ max, apesar de não se encontrarem diferenças estatísticas importantes entre os sexos, confirmando evidências apresentadas na literatura ${ }^{21}$, os homens apresentaram valores médios superiores em comparação às mulheres.

Com relação aos componentes plasmáticos de lipídios e de lipoproteínas, verificou-se que os teores de CT e da fração LDL-C são bastante similares entre os sexos; contudo, os teores de TG e de HDL-C são estatisticamente mais elevados entre os homens. As informações quanto aos níveis de PAD e PAS também revelaram diferenças estatísticas favoráveis aos homens. 


\begin{tabular}{|c|c|c|c|c|}
\hline & Mulheres $(n=41)$ & Homens $(\mathrm{n}=21)$ & Teste " "t" & $p<t$ \\
\hline $\mathrm{CCQ}$ & $0,81 \pm 0,07$ & $0,93 \pm 0,05$ & 6,987 & 0,000 \\
\hline IMC & $23,87 \pm 3,45$ & $26,81 \pm 2,41$ & 3,485 & 0,001 \\
\hline $\mathrm{VO}_{2} \max$ & $37,25 \pm 9,77$ & $42,05 \pm 9,06$ & 1,757 & 0,085 \\
\hline $\mathrm{CT}^{2}$ & $187,51 \pm 48,27$ & $204,10 \pm 42,87$ & 1,332 & 0,189 \\
\hline HDL-C & $44,57 \pm 7,71$ & $51,83 \pm 13,20$ & 2,743 & 0,008 \\
\hline LDL-C & $120,10 \pm 37,88$ & $127,48 \pm 29,03$ & 0,789 & 0,437 \\
\hline TG & $81,80 \pm 46,76$ & $136,62 \pm 63,77$ & 3,851 & 0,000 \\
\hline PAD & $76,90 \pm 10,90$ & $84,29 \pm 8,27$ & 2,727 & 0,008 \\
\hline PAS & $114,34 \pm 16,91$ & $127,57 \pm 10,08$ & 3,290 & 0,002 \\
\hline
\end{tabular}

A matriz dos coeficientes de correlação simples, incluindo informações relacionadas à CCQ, ao IMC, ao $\mathrm{VO}_{2}$ max, ao perfil lipídico-lipoprotéico e à PA, encontram-se na tabela II. A princípio, verificou-se que a essência dos coeficientes de correlação simples é bastante similar em ambos os sexos; no entanto, os valores de " $r$ " encontrados são discretamente mais elevados entre os homens.

Quanto aos níveis séricos de lipídios e de lipoproteínas, verificou-se que, em ambos os sexos, as medidas de LDL-Ce de TG foram as que produziram as correlações mais elevadas com informações associadas à CCQ. Os valores de "r" encontrados apontam para uma relação positiva e respondem por uma variação estatística em torno de $12-20 \%$ e $17-22 \%\left(r^{2} \times 100\right)$. As medidas relacionadas ao CT e ao HDL$\mathrm{C}$ não demonstraram associações que possam ser destacadas estatisticamente, sugerindo possivelmente que, nesses casos, os efeitos negativos da distribuição centrípeta da gordura corporal possam não interferir na variação de seus valores.

Os coeficientes que procuram traduzir a associação entre os níveis de PA e a CCQ foram um tanto quanto mais elevados entre os homens, explicando uma variação por volta de 20-25\%. Entre as mulheres, essa proporção diminuiu para valores próximos a 9-10\%, não alcançando significância estatística. Desse modo, pôde-se inferir que os níveis de PA entre os homens foram mais dependentes da maior concentração de gordura na região central do corpo que entre as mulheres.

Os resultados observados mediante a relação entre IMC e as variáveis sangüíneas analisadas foram bastante semelhantes aos apresentados pela CCQ. No entanto, quanto à PAD e à PAS, verificou-se que informações relacionadas ao IMC foram as que produziram associações de maior magnitude. Em valores estatísticos, em ambos os sexos, variações em torno de 25-29\% foram atribuídas às modificações observadas nos IMC, o que evidenciou o substancial impacto do sobrepeso nos níveis de PA.

Com relação às estimativas do $\mathrm{VO}_{2} \max$, enquanto as mulheres demonstraram correlações estatisticamente significativas com todos os itens de dosagem sanguiínea considerados, apontando na direção de que quanto mais elevado o nível de prática da atividade física, menor o teor de lipídios e de lipoproteínas plasmáticas, os homens apresentaram evidências nesse sentido, apenas com relação ao HDL-C. Contudo, deve-se ter alguma cautela quando da análise das diferenças sexuais encontradas, pois os valores de " $r$ " apresentados por ambos os sexos foram bastante similares, oferecendo, portanto, indicações de que a capacidade de explicação do $\mathrm{VO}_{2} \max$ na variação das dosagens sangüíneas realizadas pode ser semelhante e de alguma magnitude em ambos os sexos. Os coeficientes de correlação entre níveis de $\mathrm{PA} \mathrm{e} \mathrm{VO}_{2}$ max foram discretamente mais

Tabela II - Coeficientes de correlação simples entre relação da circunferência cintura/quadril (CCQ), índice de massa corporal (IMC), consumo máximo de oxigênio $\left(\mathrm{VO}_{2} \mathrm{max}\right)$ e níveis plasmáticos de lipídios-lipoproteínas e pressão arterial

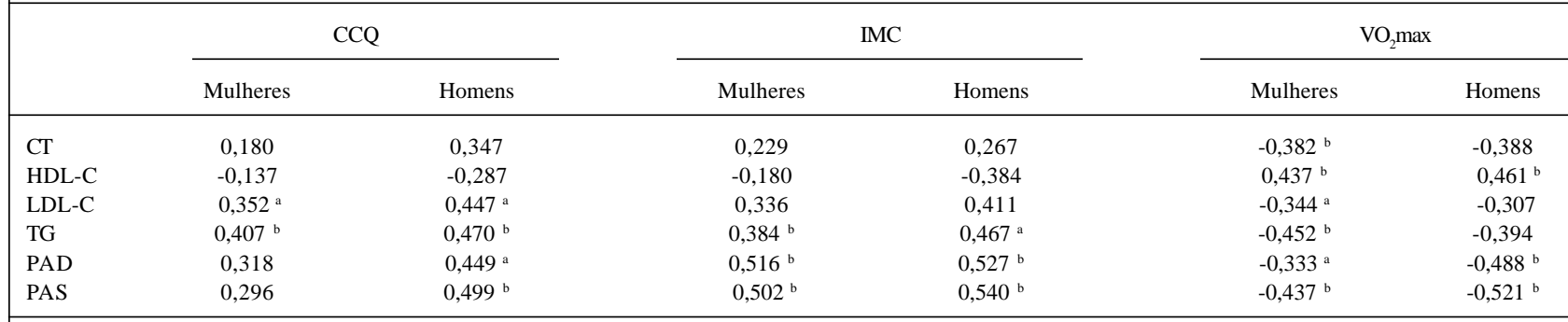

CT- colesterol total; HDL-C- lipoproteínas de alta densidade; LDL-C lipoproteínas de baixa densidade; TG- triglicérides; PAD- pressão arterial diastólica; PAS- pressão arterial sistólica; ${ }^{\text {a }} 0,05<\mathrm{p}<0,01 ;{ }^{\mathrm{b}} \mathrm{p}<0,01$. 
elevados entre os homens; porém, em ambos os sexos, os valores de " $r$ " encontrados apresentaram significância estatística.

Muito embora a magnitude e a direção dos coeficientes de correlação simples observados no presente estudo confirmam achados apresentados pela literatura ${ }^{1,2,7,9-11,22}$, a inter-relação verificada entre CCQ, IMC e $\mathrm{VO}_{2}$ max poderá confundir uma interpretação mais consistente quanto à real associação entre a distribuição anatômica da gordura corporal e níveis de PA e de lipídios-lipoproteínas plasmáticas. A magnitude da relação CCQ-IMC foi traduzida por valores positivos de " $r$ " em torno de 0,40 entre as mulheres e 0,55 entre os homens. As informações associadas à CCQ correlacionaram-se inversamente com estimativas do $\mathrm{VO}_{2} \max$, mediante valores na ordem de 0,30 e 0,40 , respectivamente.

Com base nessas evidências, recorreu-se aos procedimentos do coeficiente de correlação parcial na tentativa de determinar se as informações associadas à CCQ, que anteriormente haviam conseguido explicar alguma variação nos níveis de PA e de lipídios-lipoproteínas plasmáticas, continuavam mantendo sua influência com os efeitos do IMC e do $\mathrm{VO}_{2}$ max controlados estatisticamente (tab. III).

Enquanto a CCQ, em conjunto com outras variáveis, produziu coeficientes de correlação simples que permitem explicar variações de até $25 \%$ nos níveis de PA, ao suprimir os efeitos estatísticos atribuídos ao IMC e ao $\mathrm{VO}_{2} \max$, sua participação diminuiu para índices bastante modestos, em torno de 6\%. Quanto aos níveis de lipídios-lipoproteínas plasmáticas, ao remover os efeitos do IMC, as proporções de variações atribuídas pelo coeficiente de correlação parcial restante entre as medidas da CCQ e os teores sangüíneos de LDL-C e de TG mantiveram-se significativas estatisticamente. Confrontando com os coeficientes de correlação simples, entre os homens, os valores de $r^{2}$ x 100 demonstraram elevações de 20-22\% para 25-30\%; e, entre as mulheres, permaneceram constantes com valores próximos a 13\%. Com relação ao CT e ao HDL-C, ao controlar os efeitos do IMC, observa-se discreta redução na magnitude dos valores de "r", permanecendo sem apresentar qualquer significado estatístico.

\begin{tabular}{|c|c|c|c|c|}
\hline \multicolumn{5}{|c|}{$\begin{array}{c}\text { Tabela III - Coeficientes de correlação parcial entre relação circunfe- } \\
\text { rência cintura/quadril (CCQ) e níveis plasmáticos de lipídios- } \\
\text { lipoproteínas e pressão arterial, com os efeitos do índice de massa } \\
\text { corpórea (IMC) e do consumo máximo de oxigênio ( } \mathrm{VO}_{2} \text { max) } \\
\text { controlados }\end{array}$} \\
\hline & \multicolumn{2}{|c|}{ Controlando IMC } & \multicolumn{2}{|c|}{ Controlando $\mathrm{VO}_{2} \max$} \\
\hline & Mulheres & Homens & Mulheres & Homens \\
\hline $\mathrm{CT}$ & 0,092 & 0,240 & 0,177 & 0,305 \\
\hline HDL-C & $-0,119$ & $-0,249$ & $-0,242$ & $-0,217$ \\
\hline LDL-C & $0,325^{a}$ & $0,502^{b}$ & 0,227 & 0,202 \\
\hline TG & $0,364^{\text {a }}$ & $0,546^{\mathrm{b}}$ & 0,257 & 0,223 \\
\hline PAD & 0,243 & 0,211 & 0,271 & 0,158 \\
\hline PAS & 0,207 & 0,246 & 0,285 & 0,279 \\
\hline \multicolumn{5}{|c|}{$\begin{array}{l}\text { CT- colesterol total; HDL-C- lipoproteínas de alta densidade; LDL-C- } \\
\text { lipoproteínas de baixa densidade; TG- triglicérides; PAD- pressão arterial } \\
\text { diastólica; PAS- pressão arterial sistólica; }{ }^{\text {a }} 0,05<\mathrm{p}<0,01 ;{ }^{\text {b }} \mathrm{p}<0,01 \text {. }\end{array}$} \\
\hline
\end{tabular}

Ao inibir a participação do $\mathrm{VO}_{2} \max$, os coeficientes de correlação parcial encontrados entre CCQ e os níveis de lipídios-lipoproteínas plasmáticas apresentaram importante decréscimo de magnitude, descartando qualquer possibilidade de associação estatisticamente significativa do indicador de distribuição anatômica da gordura corporal nas dosagens sangüíneas realizadas. Enquanto as medidas da CCQ, mediante o coeficiente de correlação simples, responderam por entre $20-22 \%$ e $12-17 \%$ da variação dos teores de LDL-C e de TGem homens e mulheres, respectivamente, ao isolar os efeitos das estimativas do $\mathrm{VO}_{2} \max$, a contribuição relativa se reduziu a não mais que $4-5 \%$ e $5-7 \%$.

\section{Discussão}

Nossos resultados sugerem que, após a correção pelo IMC, permanece uma significativa correlação parcial entre CCQ e níveis séricos de LDL-C e de TG, enfatizando a relativa importância da maior adiposidade na região central do corpo nos teores plasmáticos de lipídios e de lipoproteínas, independentemente, da quantidade de gordura corporal. Contudo, quando da tentativa de controlar, estatisticamente, os efeitos associados ao $\mathrm{VO}_{2}$ max, verificou-se que a participação da CCQ nos componentes plasmáticos analisados tenderam a diminuir, o que permite especular quanto à importante participação da prática da atividade física na relação entre distribuição anatômica da gordura corporal-perfil plasmático de lipídios-lipoproteínas.

Ao tratar os dados relacionados à PA, quando do isolamento em valores estatísticos, tanto das informações associadas ao IMC como ao $\mathrm{VO}_{2}$ max, constatou-se que os coeficientes de correlação CCQ-PAD e CCQ-PAS tenderam a enfraquecer. Esses resultados apontam para a possibilidade da relação distribuição anatômica da gordura corporalPA sofrer interferência do nível de prática da atividade física e da quantidade de gordura corporal.

Os mecanismos mediante os quais um predomínio da gordura localizada na região central do corpo possa comprometer os níveis de lipídios-lipoproteínas plasmáticas e de PA parecem não estar totalmente esclarecidos; porém, algumas possibilidades são discutidas na literatura. Larsson e $\operatorname{col}^{23}$ têm sugerido que a disposição centrípeta da gordura corporal pode representar um aumento no tamanho e/ou no número das células adiposas intra-abdominal ou visceral, metabolicamente, mais ativas. Essas células adiposas liberam maiores quantidades de ácido graxo livre na circulação portal, expondo dessa maneira, o fígado e os tecidos periféricos à concentração mais elevada desse componente lipídico. Essa situação deverá induzir a reduções na extração hepática da insulina, devido à diminuição do número de receptores que, por sua vez, leva a hiperinsulinemia periférica. Como conseqüência, vários outros processos metabólicos são afetados, comprometendo a PA e o perfil plasmático dos lipídios e das lipoproteínas.

Para Kissebah e Peiris ${ }^{5}$, uma outra possibilidade que pode justificar os níveis adversos de PA e de lipídioslipoproteínas plasmáticas, em função da localização predo- 
minantemente centrípeta da gordura corporal, está associada ao controle neuro-endócrino. A maior concentração de gordura na região central do corpo resulta em adaptações hormonais relacionadas ao aumento dos níveis de cortisol e diminuição nas secreções de esteróides sexuais. Esse novo perfil hormonal deverá afetar o processo de síntese dos carboidratos, aumentando a predisposição às complicações endócrinas e metabólicas.

Nessa mesma direção, Freedman e $\mathrm{col}^{8}$ advogam que os efeitos da secreção dos hormônios esteróides e a densidade local de seus receptores decidem duas características sexuais específicas de distribuição regional da gordura corporal: a) homens com maiores proporções de tecido adiposo na região central e mulheres nas extremidades; e b) mulheres com maiores proporções do tecido adiposo na região subcutânea e homens nas regiões viscerais. O menor acúmulo de gordura visceral nas mulheres ocorre em conseqüência dos efeitos da progesterona na ação do cortisol. Nos homens, a concentração mais elevada de gordura visceral associa-se a reduções nos níveis de testosterona livre e ao concomitante acréscimo na secreção de cortisol. $O$ cortisol estimula a mobilização e o maior acúmulo de lipídios mediante o aumento na atividade da lipoproteína lipase, caracterizada como principal enzima mediadora do consumo de triglicerídios pelos tecidos. Se bem que homens jovens e magros podem apresentar menores quantidades de gordura nos depósitos abdominais e viscerais. Nestes casos, a secreção de testosterona é alta, acentuando seus efeitos sobre a lipólise e se contrapondo à ação do cortisol.

Segundo Björntorp ${ }^{4}$, o estresse emocional também pode se configurar como mais um fator a desencadear aumentos na secreção de cortisol, contribuindo, portanto, para uma maior concentração de gordura nas regiões viscerais.

Quanto às evidências de que os valores estimados do $\mathrm{VO}_{2}$ max possam atenuar os efeitos deletérios do padrão de distribuição de gordura centrípeto nos níveis de PA e de LDL-C e TG plasmáticos, duas situações podem ser consideradas: $1^{\mathrm{a}}$, os efeitos positivos dos programas regulares de exercícios físicos na redução dos níveis de PA e de lipídioslipoproteínas plasmáticas; $2^{\mathrm{a}}$, as respostas dos diferentes depósitos de gordura regional associada à prática de exercícios aeróbicos.

Nos últimos anos, importantes estudos, envolvendo delineamentos experimentais extremamente bem elaborados, têm demonstrado que indivíduos submetidos a programas regulares de exercícios físicos apresentam alterações favoráveis quanto à $\mathrm{PA}^{24,25}$ e ao perfil plasmático de lipídioslipoproteínas ${ }^{13,26}$.

Os mecanismos responsáveis pela redução nos níveis de PA e de lipídios-lipoproteínas plasmáticas proveniente da prática de exercícios físicos ainda são bastante especulativos. No entanto, Haskell ${ }^{12}$ sugere que o aumento na atividade da lipoproteína lipase no músculo esquelético e/ou no tecido adiposo durante a realização dos exercícios físicos e por várias horas pós-esforço, associado ao possível decréscimo da síntese hepática dos triglicerídios, possam ser ajustes metabólicos que favorecem uma menor concentração de lipídios plasmáticos. Com relação à PA, Marceau ${ }^{24}$ chama a atenção para algumas adaptações fisiológicas que podem auxiliar na redução de seus valores: diminuição nos níveis plasmáticos de noradrenalina, aumento na circulação de substâncias vasodilatadoras, melhora no estado hiperinsulínico e redução da atividade neural simpática.

Estudos prévios têm verificado que a realização de exercícios físicos predominantemente aeróbicos, poderão induzir a maior mobilização da gordura localizada na região abdominal, notadamente entre os homens ${ }^{27}$, além de estabelecer mecanismos de proteção contra o maior acúmulo de gordura visceral, próprio das idades mais avançadas ${ }^{28}$. Essa adaptação favorável do perfil de adiposidade pode ser atribuída a variação regional associada às atividades metabólicas do tecido adiposo, traduzida por atividades lipolíticas mais elevadas nos depósitos de gordura da região central do corpo em comparação com as extremidades.

Diante da perspectiva de que o excesso de gordura na região central do corpo está associado aos dois principais fatores de risco biológicos predisponentes ao aparecimento e desenvolvimento das doenças cardiovasculares ${ }^{29}$, a mobilização preferencial da gordura localizada nessa região mediante a prática de exercícios aeróbicos deverá se caracterizar como mais um importante motivo para a inclusão da atividade física nos programas de prevenção e reabilitação das coronariopatias.

Concluindo, os resultados encontrados no estudo sugerem que, se por um lado, independentemente da quantidade de gordura corporal, a disposição centrípeta da gordura pode contribuir para o aparecimento de hipertrigliceridemia, comprometer o metabolismo das lipoproteínas plasmáticas e dificultar a manutenção da PA em níveis satisfatórios; por outro, níveis mais elevados de prática da atividade física deverão minimizar eventuais distúrbios associados ao perfil lipídicolipoprotéico e à PA que acompanham a maior concentração de gordura na região central do corpo.

\section{Referências}

1. Barakat HA, Burton DS, Carpenter JW et al - Body fat distribution, plasma lipoproteins and the risk of coronary heart disease of male subjects. Int J Obes 1988; 12: 473-80.

2. Després JP, Moorjanis S, Lupien PJ et al - Regional distribution of body fat, plasma lipoproteins and cardiovascular disease. Arteriosclerosis 1990; 10: 497-511.

3. Hauner H, Stangl K, Schmatz C et al - Body fat distribution in men with angiographically confirmed coronary artery disease. Atherosclerosis 1990; 85 : 203-10.

4. Björntorp P - Metabolic implications of body fat distribution. Diabetes Care 1991; 14: 1132-43.

5. Kissebah AH, Peiris AN - Bilogy of regional body fat distribution: relationships to non-insulin-dependent diabetes mellitus. Diabetes Metab Rev 1989; 5: 83-109. 
6. Després JP, Tremblay A, Theriault $\mathrm{G}$ et al - Relationship between body fatness, adipose tissue distribution and blood pressure in men and women. J Clin Epidemiol 1988; 41: 889-97.

7. Folin M, Contiero E - Relationship between subcutaneous fat distribution and serum lipids and blood pressures in Italian men. Am J Human Biol 1994; 4: 457-63.

8. Freedman DS, Jacobsen J, Bauboria K et al - Body fat distribution and male/female differences in lipids and lipoproteins. Circulation 1990; 81: 1498-506.

9. Jakicic JM, Donnelly JE, Jawad AF et al - Association between blood lipids an different measures of body fat distribution: effects of BMI and age. Int J Obes Relat Metab Disord 1993; 17: 131-7.

10. Anderson AJ, Sobocinski KA, Freedman DS et al - Body fat distribution, plasma lipids, and lipoproteins. Arteriosclerosis 1988; 8: 88-94.

11. Foster CJ, Weinsier RL, Birch R et al - Obesity and serum lipids: an evaluation of the relative contribution of body fat and fat distribution to lipid levels. Int J Obes 1987; 11: 151-62.

12. Haskell WL - The influence of exercise on the concentrations of triglyceride and cholesterol in human plasma. Exerc Sport Sci Rev 1984; 12: 205-44.

13. Kokkinos PF - Cardiorespiratory fitness and coronary heart disease risk factor association in women. J Am Coll Cardiol 1995; 26: 358-64

14. Friedewald WT, Levy RI, Frederickson DS - Estimation of the concentration of low density lipoprotein cholesterol in plasma, without use of the preparative ultracentrifuge. Clin Chem 1972; 18: 499-502.

15. Gordon CC, Chumlea WC, Roche AP et al - Stature, recumbent length, and weight. In: Lohman TG, Roche AF, Martorel R. ed - Anthropometric Standardization Reference Manual. Champaign, Illinois: Human Kinetics Books, 1988: 39 54.

16. Callaway CW, Chumlea WC, Bouchard C et al - Circunferences. In: Lohman TG, Roche AF, Martorel R ed - Anthropometric Standardization Reference Manual. Champaign, Illinois: Human Kinetics Books, 1988: 39-54

17. Bruce RA, Kusumi F, Hosmer D - Maximal oxygen intake and nomographic as- sessment of functional aerobic impairment in cardiovascular disease. Am Heart J 1973; 85: 545-62

18. American College of Sports Medicine - Guidelines for Exercise Testing and Prescription. $5^{\text {th }}$ ed. Philadelphia: Williams \& Wilkins, 1995.

19. Foster C, Jackson AS, Pollock ML et al - Generalized equations for predicting functional capacity from treadmill performance. Am Heart J 1984; 107: 1229-34.

20. SAS. Sas/Stat User's Guide - Version 3.0. $4^{\text {th }}$ ed. Cary: SAS Institute, 1990.

21. McArdle WD, Katch FI, Katch VL-Essentials of Exercise Physiology. Philadelphia: Lea \& Febiger, 1994.

22. Kissebah AH, Evans DJ, Peiris A et al - Endocrine characteristics in regional obesities: role of sex steroids. In: Vague J. ed - Metabolic Complications of Human Obesities. Amsterdam: Elsevier, 1985: 115-30.

23. Larsson B, Svardsudd K, Welin L et al - Abdominal adipose tissue distribution, obesity, and risk of cardiovascular disease and death: 13 year follow up of participants in the study of men born in 1913. Br Med J 1984; 288: 1401-4.

24. Marceau M-Effects of different training intensities on 24-hours blood pressure in hipertensive subjects. Circulation 1993; 88: 2803-11.

25. Pescatello LS - Short-term effects of dynamic exercise on arterial blood pressure. Circulation 1991; 83: 1557-61.

26. Gordon PM, Goss FL, Visich PS et al - The acute effects of exercise intensity on HDL-C metabolism. Med Sci Sports Exerc 1994; 26: 671-7.

27. Després JP, Pouliot MG, Moorjani S et al - Loss of abdominal fat and metabolic response to exercise training in obese women. Am J Physiol 1991; 261: E159E67.

28. Hohrt WM, Malley MT, Dalsky GPet al - Body composition of healthy sedentary and trained, young and older men and women. Med Sci Sports Exerc 1992; 24: $832-7$

29. Terry RB, Stefanick ML, Haskell W et al-Contributions of regional adipose tissue depots to plasma lipoprotein concentrations in overweight men and women: possible protective effects of thigh fat. Metabolism 1991; 40: 733-40. 